Chem. Ber. 113, 2100-2109 (1980)

\title{
Transacylierungen mit Acylderivaten des 4-Pyridons
}

\author{
Franz Effenberger* und Eberhard Bessey ${ }^{1)}$ \\ Institut für Organische Chemie der Universität Stuttgart, \\ Pfaffenwaldring 55, D-7000 Stuttgart 80
}

Eingegangen am 8. Oktober 1979

Mit N-Acyl-4-pyridonen (2a, b) sowie mit 4-(Benzoyloxy)pyridin (3a) werden in Methylenchlorid oder Chloroform aliphatische und aromatische Amine sowie Thiole glatt zu den Amiden (4-6) und Thioestern $(7,8)$ acyliert. Primäre und sekundäre Alkohole reagieren langsamer, tertButylalkohol nur noch mit $\mathbf{3 a}$ unter Basenkatalyse sowie mit den reaktiveren $N$-(Trihalogenacetyl)-4-pyridonen $3 \mathbf{b}$, c zu den tert-Butylestern 12. N-Acetyl-4-pyridon (2a) besitzt ein deutlich höheres Acylierungspotential als $N$-Acetylimidazol (13a).

\section{Transacylations with Acyl Derivatives of 4-Pyridones}

Aliphatic and aromatic amines as well as thiols react with $N$-acyl-4-pyridones $(\mathbf{2} \mathbf{a}, \mathbf{b})$ and with 4-(benzoyloxy)pyridine (3a) in methylene chloride or chloroform to give the $N$-substituted amides $4-6$ and the thiol esters 7 and 8 , respectively, with very good yields. Primary and secondary aicohols react more slowly, tert-butyl alcohol only with $\mathbf{3 a}$ to the tert-butyl esters 12 under basic catalysis or with the much more reactive $N$-(trihaloacetyl)-4-pyridones $3 \mathbf{b}, \mathbf{c}$. $N$-Acetyl-4-pyridone (2a) possesses a much higher acylation potential than $N$-acetylimidazole (13a).

In der vorstehenden Mitteilung haben wir über Acylierungen des 4-Pyridons (1) zu $N$-Acyl-4pyridonen 2 bzw. 4-(Acyloxy)pyridinen 3 berichtet ${ }^{2)}$.<smiles>[R]C(=O)Oc1ccncc1</smiles>

Überraschend war, daß sich beim Lösen von 2 bzw. 3 in Chloroform, Methylenchlorid, Acetonitril u. a. rasch ein Gleichgewicht $2=3$ einstellt, dessen Lage hauptsächlich vom Acylsubstituenten $\mathbf{R}$, aber auch von der Polarität des Lösungsmittels und der Temperatur abhängt ${ }^{2}$ ). Die unter milden Reaktionsbedingungen verlaufende Acylwanderung veranlaßte uns, die Acylderivate des 4-Pyridons auf ihre Verwendbarkeit als Transacylierungsagentien zu untersuchen.

Vergleicht man die acylierten Pyridone mit ähnlichen Acylierungsagentien, so sind die $N$ Acylderivate 2 mit den Azoliden ${ }^{3)}$, die $O$-Acylverbindungen 3 mit aktivierten Estern (z. B. $p$ Nitrophenylestern) verwandt. Für ihre Anwendung als Transacylierungsmittel war es deshalb zunächst ohne Bedeutung, ob Verbindungen des Typs 2 oder 3 vorliegen; außerdem konnte man davon ausgehen, daß über die rasche Einstellung des Gleichgewichts $2 \rightleftharpoons 3$ das reaktivere Acylierungsagens im Reaktionsmedium ausreichend schnell nachgebildet wird. 
2-(Acyloxy)pyridine wurden schon gelegentlich als Acylierungsmittel eingesetzt ${ }^{4,5)}$, häufiger wird jedoch 2-Hydroxypyridin als Katalysator bei Acylierungsreaktionen verwendet ${ }^{6}$ ). Die Ausbildung energetisch günstiger Sechsringübergangszustände ${ }^{7)}$ bzw. Zwischenverbindungen wird häufig für die Eignung des 2-Pyridons als Katalysator bei diesen Acylierungsreaktionen angegeben, eine strukturelle Voraussetzung, die für 4-Pyridon (1) nicht zutrifft.

Neben der Untersuchung der allgemeinen Anwendungsbreite der Verbindungen 2 bzw. 3 als Transacylierungsmittel interessierten vor allem auch die Unterschiede dieser Agentien gegenüber den erwähnten Azoliden und aktivierten Estern. Präparative Vorteile bei Acylierungen mit 2 bzw. 3 sollten sich aus der Schwerlöslichkeit von 1 in unpolaren organischen Lösungsmitteln ergeben. Bei Wahl eines geeigneten Lösungsmittels wird 1, das im Verlauf der Umsetzung von Nucleophilen mit 2 bzw. 3 frei wird, dem Reaktionssystem kontinuierlich entzogen, was sich günstig auf das Acylierungspotential von 2 bzw. 3 auswirken sollte.

\section{Acylierung von Aminen}

Bei Umsetzung von aliphatischen bzw. aromatischen Aminen mit $N$-Acetyl-4pyridon (2a) ${ }^{2)}$ 4-(Benzoyloxy)pyridin (3 a ${ }^{2)}$ bzw. $N$-Formyl-4-pyridon (2 b), das - bedingt durch seine Instabilität - in reiner Form nur schwer zugänglich ist ${ }^{8)}$, entstehen

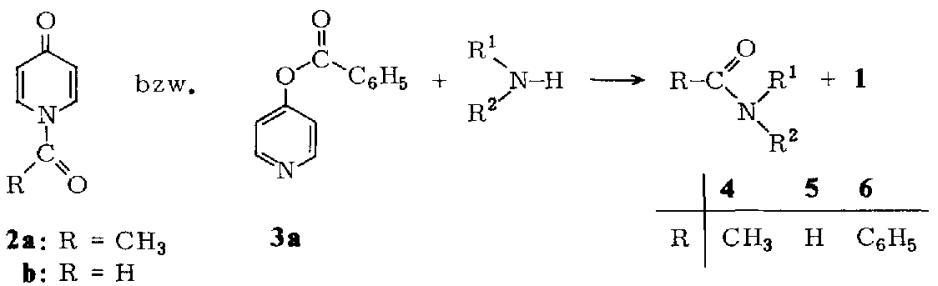

\begin{tabular}{c|llll}
$\mathbf{4 - 6}$ & a & b & c & d \\
\hline $\mathrm{R}^{2}$ & $\mathrm{H}$ & $\stackrel{\mathrm{H}}{\mathrm{l}} \mathrm{H}$ & $\mathrm{H}$ \\
$\mathrm{R}^{1}$ & $\mathrm{C}_{6} \mathrm{H}_{11}$ & & $\mathrm{HOCH}_{2} \mathrm{CH}_{2}$ & $\mathrm{C}_{6} \mathrm{H}_{5}$
\end{tabular}

Tab. 1. Acylierung von Aminen mit den acylierten Pyridonen 2a, b und 3a in Methylenchlorid bei Raumtemperatur

\begin{tabular}{|c|c|c|c|c|c|c|}
\hline $\begin{array}{l}\text { Acylie- } \\
\text { rungs- } \\
\text { mittel }\end{array}$ & $\mathrm{R}^{1}$ Ami & $\mathrm{R}^{2}$ & $\begin{array}{l}\text { Reaktions- } \\
\text { zeit }\end{array}$ & $\begin{array}{l}4 \\
5 \\
6\end{array}$ & $\begin{array}{c}\text { Produkt } \\
\text {-acetamid } \\
\text {-formamid } \\
\text {-benzamid }\end{array}$ & $\begin{array}{c}\text { Ausb. } \\
(\%)\end{array}$ \\
\hline $\left.\begin{array}{l}2 \mathbf{a} \\
2 \mathbf{b} \\
3 \mathbf{a}\end{array}\right\}$ & $\mathrm{C}_{6} \mathrm{H}_{11}$ & $\mathrm{H}$ & $5 \mathrm{~min}$ & $\begin{array}{l}4 a \\
5 \mathrm{a} \\
6 \mathrm{a}\end{array}$ & $N$-Cyclohexyl- & $\begin{array}{l}86 \\
87 \\
89\end{array}$ \\
\hline $\left.\begin{array}{l}2 \mathbf{a} \\
2 \mathbf{b} \\
3 \mathbf{a}\end{array}\right\}$ & $-\left[\mathrm{CH}_{2}\right]_{5}-$ & & $5 \mathrm{~min}$ & $\begin{array}{l}4 b \\
5 b \\
6 b\end{array}$ & $N, N$-Pentamethylen- & $\begin{array}{l}95 \\
84 \\
93\end{array}$ \\
\hline $\left.\begin{array}{l}\mathbf{2} \mathbf{a} \\
\mathbf{2} \mathbf{b}\end{array}\right\}$ & $\mathrm{HOCH}_{2} \mathrm{CH}_{2}$ & $\mathbf{H}$ & $5 \mathrm{~min}$ & $\begin{array}{l}4 c \\
5 c\end{array}$ & $N$-(2-Hydroxyethyl)- & $\begin{array}{l}85 \\
80\end{array}$ \\
\hline $\left.\begin{array}{l}2 \mathbf{a} \\
2 \mathbf{b} \\
3 \mathbf{a}\end{array}\right\}$ & $\mathrm{C}_{6} \mathrm{H}_{5}$ & $\mathrm{H}$ & $\begin{array}{l}120 \mathrm{~min} \\
30 \mathrm{~min} \\
10 \mathrm{~h}\end{array}$ & $\begin{array}{l}4 d \\
5 d \\
6 d\end{array}$ & $N$-Phenyl- & $\begin{array}{l}81 \\
83 \\
85\end{array}$ \\
\hline
\end{tabular}


schon bei Raumtemperatur - zum Teil in exothermer Reaktion - die $N$-substituierten Säureamide 4- 6 in sehr guten Ausbeuten.

Von den bifunktionellen Verbindungen konnte Ethanolamin ausschließlich an der Aminogruppe - ohne nachweisbare Esterbildung - acyliert werden. Bei der Acylierung des $m$-Aminophenols erfolgt unter den angewandten Bedingungen sowohl $N$-als auch $O$-Acylierung; mit der zweifach molaren Menge 2 a erhielten wir in 91 proz. Ausbeute 3-Acetoxy- $N$-acetylanilin (s. Tab. 4).

\section{Acylierung von Thiolen ${ }^{3 b)}$}

Auch Mercaptane und Thiophenole reagieren mit 2a und 3a in sehr guten Ausbeuten $(85-91 \%)$ unter Transacylierung zu den Carbonsäure-thiolestern 7 bzw. 8. Die Umsetzungen wurden in Chloroform bei Raumtemperatur durchgeführt.

$$
\begin{aligned}
& 2 \mathrm{a} \mathrm{bzw} \cdot 3 \mathrm{a}+\mathrm{R}^{1}-\mathrm{SH} \longrightarrow \mathrm{R}-\mathrm{C}_{\mathrm{S}-\mathrm{R}^{1}}^{\mathrm{O}}+\mathbf{1} \\
& \begin{array}{ll|lll}
\text { 7: } \mathrm{R}=\mathrm{CH}_{3} & & \mathbf{a} & \text { b } & \text { c } \\
\text { 8: } \mathrm{R}=\mathrm{C}_{6} \mathrm{H}_{5} & \mathrm{R}^{1} & \mathrm{n}-\mathrm{C}_{4} \mathrm{H}_{9} & \mathrm{C}_{6} \mathrm{H}_{5} \mathrm{CH}_{2} & \mathrm{C}_{6} \mathrm{H}_{5}
\end{array}
\end{aligned}
$$

\begin{tabular}{|c|c|c|c|c|c|}
\hline $\begin{array}{l}\text { Acylie- } \\
\text { rungs- } \\
\text { mittel }\end{array}$ & $\begin{array}{l}\text { Thiol } \\
\mathrm{R}^{1}\end{array}$ & $\begin{array}{c}\text { Reaktions- } \\
\text { zeit }\end{array}$ & $\begin{array}{l}7 \\
8\end{array}$ & $\begin{array}{c}\text { Produkt } \\
\text { Thioessigsäure- } \\
\text { Thiobenzoesäure- }\end{array}$ & $\begin{array}{c}\text { Ausb. } \\
(\%)\end{array}$ \\
\hline $\left.\begin{array}{l}2 \mathrm{a} \\
3 \mathrm{a}\end{array}\right\}$ & $\mathrm{n}-\mathrm{C}_{4} \mathrm{H}_{9}$ & $\begin{array}{l}5 \mathrm{~min} \\
10 \mathrm{~h}\end{array}$ & $\begin{array}{l}7 \mathbf{a} \\
8 \mathbf{a}\end{array}$ & -S-butylester & $\begin{array}{l}85 \\
89\end{array}$ \\
\hline $\left.\begin{array}{l}2 \mathbf{a} \\
3 \mathbf{a}\end{array}\right\}$ & $\mathrm{C}_{6} \mathrm{H}_{5} \mathrm{CH}_{2}$ & $\begin{array}{l}5 \mathrm{~min} \\
10 \mathrm{~h}\end{array}$ & $\begin{array}{l}7 \mathbf{b} \\
8 \mathbf{b}\end{array}$ & -S-benzylester & $\begin{array}{l}88 \\
91\end{array}$ \\
\hline $\left.\begin{array}{l}2 \mathbf{a} \\
3 \mathbf{a}\end{array}\right\}$ & $\mathrm{C}_{6} \mathrm{H}_{5}$ & $\begin{array}{l}5 \text { min } \\
1 \mathrm{~h}\end{array}$ & $\begin{array}{l}7 c \\
8 c\end{array}$ & -S-phenylester & $\begin{array}{l}87 \\
89\end{array}$ \\
\hline
\end{tabular}

Tab. 2. Acylierung von Thiolen mit $\mathbf{2 a}$ bzw. $\mathbf{3 a}$ in Chloroform bei Raumtemperatur

\section{Acylierung von Alkoholen und Phenolen}

Erwartungsgemäß lassen sich Alkohole infolge ihrer geringeren Nucleophilie nicht so leicht acylieren wie Amine oder Thiole. Auch innerhalb der Klasse der Alkohole ist eine deutliche Abstufung der Reaktivität vorhanden, so daß die Acylierung primärer, sekundärer und tertiärer Alkohole unterschiedliche Reaktionsbedingungen erforderlich macht.

Primäre Alkohole und Phenole reagieren mit der Acetylverbindung 2a noch bei Raumtemperatur in Methylenchlorid in guten Ausbeuten zu den Estern 9, wenn die Reaktionszeit auf $12 \mathrm{~h}$ verlängert wird; bei der Veresterung mit dem weniger reaktiven Benzoylderivat 3a sind dagegen Temperaturen um $40^{\circ} \mathrm{C}$ erforderlich, um nach gleicher Reaktionsdauer die Benzoesäureester 10 in guten Ausbeuten darzustellen. Die Acylierung sekundärer Alkohole erfordert zur Erzielung guter Ausbeuten in annehmbaren Reaktionszeiten sowohl mit 2 a als auch mit 3 a eine Reaktionstemperatur von $40^{\circ} \mathrm{C}$. 


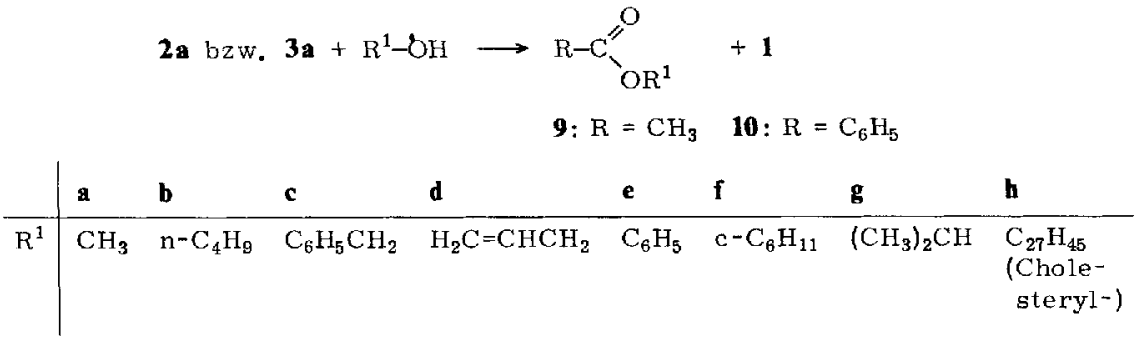

Tab. 3. Acylierung primärer und sekundärer Alkohole mit 2a bzw. 3a in Methylenchlorid

\begin{tabular}{|c|c|c|c|c|c|c|}
\hline $\begin{array}{l}\text { Acylie- } \\
\text { rungs- } \\
\text { mittel }\end{array}$ & $\begin{array}{l}\text { Alkohol } \\
\mathbf{R}^{1}\end{array}$ & $\begin{array}{l}\text { Real } \\
\text { zeit } \\
h\end{array}$ & $\begin{array}{l}\text { tions- } \\
\text { temp. } \\
{ }^{\circ} \mathrm{C}\end{array}$ & $\begin{array}{r}9 \\
10\end{array}$ & $\begin{array}{c}\text { Produkt } \\
\text { Essigsäure- } \\
\text { Benzoesäure- }\end{array}$ & $\underset{(\%)}{\text { Ausb }}$ \\
\hline $\left.\begin{array}{l}\mathbf{2} \mathbf{a} \\
\mathbf{3} \mathbf{a}\end{array}\right\}$ & $\mathrm{CH}_{3}$ & 12 & $\begin{array}{l}20 \\
40\end{array}$ & $\begin{array}{r}9 a \\
10 a\end{array}$ & -methylester & $\begin{array}{r}100 \\
84\end{array}$ \\
\hline $\left.\begin{array}{l}2 \mathbf{a} \\
3 \mathbf{a}\end{array}\right\}$ & $\mathrm{n}-\mathrm{C}_{4} \mathrm{H}_{9}$ & 12 & $\begin{array}{l}20 \\
40\end{array}$ & $\begin{array}{r}9 b \\
10 b\end{array}$ & -butylester & $\begin{array}{l}91 \\
84\end{array}$ \\
\hline $\left.\begin{array}{l}2 \mathbf{a} \\
3 \mathbf{a}\end{array}\right\}$ & $\mathrm{C}_{6} \mathrm{H}_{5} \mathrm{CH}_{2}$ & 12 & $\begin{array}{l}20 \\
40\end{array}$ & $\begin{array}{r}9 \mathrm{c} \\
10 \mathrm{c}\end{array}$ & -benzylester & $\begin{array}{l}87 \\
88\end{array}$ \\
\hline $\left.\begin{array}{l}\mathbf{2 a} \\
\mathbf{3 a}\end{array}\right\}$ & $\mathrm{H}_{2} \mathrm{C}=\mathrm{CH}-\mathrm{CH}_{2}$ & 12 & $\begin{array}{l}20 \\
40\end{array}$ & $\begin{array}{r}9 \mathrm{~d} \\
10 \mathrm{~d}\end{array}$ & -allylester & $\begin{array}{l}91 \\
79\end{array}$ \\
\hline $\left.\begin{array}{l}2 \mathbf{a} \\
\mathbf{3} \mathbf{a}\end{array}\right\}$ & $\mathrm{C}_{6} \mathrm{H}_{5}$ & 12 & $\begin{array}{l}20 \\
40\end{array}$ & $\begin{array}{r}9 \mathrm{e} \\
10 \mathrm{e}\end{array}$ & -phenylester & $\begin{array}{l}86 \\
88\end{array}$ \\
\hline $\left.\begin{array}{l}\mathbf{2} \mathbf{a} \\
\mathbf{3} \mathbf{a}\end{array}\right\}$ & $c-\mathrm{C}_{6} \mathrm{H}_{11}$ & $\begin{array}{l}1 \\
2\end{array}$ & 40 & $\begin{array}{r}9 \mathrm{f} \\
10 \mathrm{f}\end{array}$ & -cyclohexylester & $\begin{array}{l}87 \\
81\end{array}$ \\
\hline $\left.\begin{array}{l}2 a \\
3 a\end{array}\right\}$ & $\left(\mathrm{CH}_{3}\right)_{2} \mathrm{CH}$ & $\begin{array}{l}4 \\
1 \\
3\end{array}$ & $\begin{array}{l}20 \\
40 \\
40\end{array}$ & $\begin{array}{r}9 \mathrm{~g} \\
9 \mathrm{~g} \\
10 \mathrm{~g}\end{array}$ & -isopropylester & $\begin{array}{r}76 \\
100 \\
85\end{array}$ \\
\hline $\left.\begin{array}{l}2 \mathbf{a} \\
3 \mathbf{a}\end{array}\right\}$ & Cholesterin & $\begin{array}{l}3 \\
5\end{array}$ & 40 & $\begin{array}{r}9 \mathrm{~h} \\
10 \mathrm{~h}\end{array}$ & -cholesterylester & $\begin{array}{l}96 \\
89\end{array}$ \\
\hline
\end{tabular}

tert-Butylalkohol reagiert mit 2 a auch beim Erwärmen auf $40^{\circ} \mathrm{C}$ nicht in nennenswerten Ausbeuten zum gewünschten tert-Butylester $12(\mathrm{X}=\mathrm{H})$. In siedendem 1,2Dichlorethan (Sdp. $83^{\circ} \mathrm{C}$ ) konnten zwar nach 3 Tagen $35 \%$ Essigsäure-tert-butylester gaschromatographisch nachgewiesen werden, die Hauptmenge $\mathbf{2 a}$ reagiert jedoch bei dieser Temperatur irreversibel zu N-(4-Pyridyl)-4-pyridon (11), eine Reaktion, die analog zu der des 4-Chlorpyridins" als nucleophiler Angriff des 4-Pyridons (1) an dem mit 2 a im Gleichgewicht vorhandenen 4-Acetoxypyridin gedeutet werden muß.

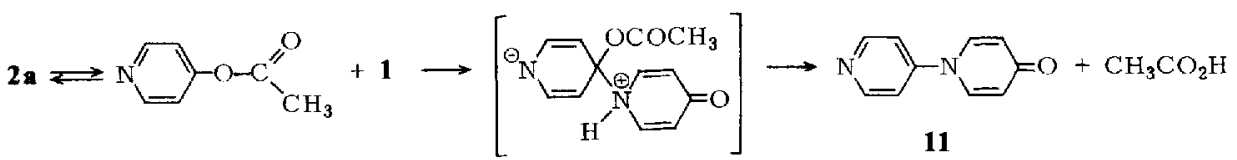

Die Acylierung tertiärer Alkohole unter milden Bedingungen und in guten Ausbeuten kann jedoch einmal durch Erhöhung der Reaktivität des Acylierungsagens und zum anderen durch Erhöhung der Nucleophilie des tertiären Alkohols - z. B. durch Basenkatalyse - erreicht werden. So erhielten wir bei Umsetzungen von 4-(Trichloracetoxy)- 
(3 b) und 4-(Trifluoracetoxy)pyridin (3c) mit tert-Butylalkohol in Methylenchlorid bei Raumtemperatur nach $3 \mathrm{~h}$ die tert-Butylester $12 \mathrm{~b}$ und c mit 45 bzw. 86\% Ausbeute.<smiles>[Y3]C(=O)Oc1ccncc1</smiles>

3<smiles>CC(C)(C)[14C]C(=O)OC(C)(C)C</smiles>

12

$$
\text { b: } X=C 1, \mathfrak{c}: X=F
$$

Über die Formylierung von Alkoholen mit $N$-Formyl-4-pyridon (2b), das als besonders reaktives Acylierungsagens ebenfalls gut mit tertiären Alkoholen reagiert, berichten wir in der nachstehenden Mitteilung ${ }^{8)}$.

Die Möglichkeit einer Basenkatalyse haben wir bei der Benzoylierung von tertButylalkohol mit 3a untersucht; in Gegenwart katalytischer Mengen Natrium-tertbutylat bildet sich Benzoesäure-tert-butylester $\left(\mathbf{1 0}, \mathrm{R}^{\mathbf{1}}=\mathrm{C}\left(\mathrm{CH}_{3}\right)_{3}\right)$ schon bei Raumtemperatur nach 4-tägiger Reaktionsdauer in einer Ausbeute von $90 \%$.

\section{Reaktivitätsvergleich acylierter Pyridone mit entsprechenden Imidazoliden}

Bei den vorstehend beschriebenen Acylierungsreaktionen kann man aufgrund der angewandten Reaktionsbedingungen davon ausgehen, daß sie als Zweistufenreaktion über eine tetraedrische Zwischenverbindung ${ }^{10)}$ ablaufen. Der erste Reaktionsschritt die Addition an die Carbonylgruppe - wird hauptsächlich durch die Nucleophilie der $\mathrm{zu}$ acylierenden Verbindung bestimmt, der zweite im wesentlichen durch die Austrittstendenz der Abgangsgruppe des Acylierungsmittels.

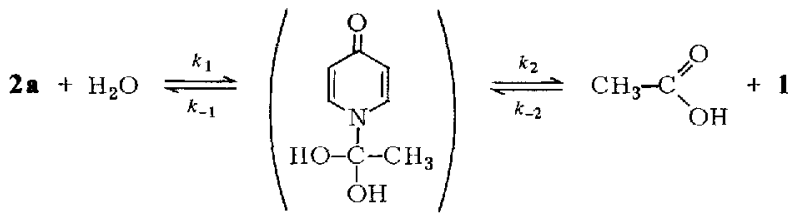

Zur Ermittlung der Reaktivität im neutralen Medium haben wir die Halbwertszeit der Hydrolyse von $N$-Acetyl-4-pyridon (2a) und 1-Acetylimidazol (13a) bestimmt. Die hydrolytische Spaltung von $\mathbf{2 a}$ wurde analog zur Ermittlung der Hydrolysegeschwindigkeit von $13 \mathbf{a}^{11)}$ konduktometrisch über die Bildung der Essigsäure verfolgt, und zwar in einem Überschuß von Wasser als Reaktion „pseudo-erster Ordnung“. Damit wird die Halbwertszeit $\tau_{1 / 2}$ unabhängig von der Konzentration des eingesetzten $2 \mathbf{a}$ und stellt ein gutes Maß für die Reaktivität dar. Für die Neutralhydrolyse von $\mathbf{2}$ a in Leitfähigkeitswasser bei $23^{\circ} \mathrm{C}$ wurde für $k^{\prime}=0.163 \mathrm{~min}^{-1}$ und damit eine Halbwertszeit von $4.3 \mathrm{~min}$ ermittelt, für $13 \mathrm{a}$ lauten die Werte $k^{\prime}=0.0132 \mathrm{~min}^{-1}$ und $\tau_{1 / 2}=50 \mathrm{~min} . N$ Acetyl-4-pyridon (2a) reagiert demnach mit Wasser in neutralem Medium ca. zehnmal schneller als 1-Acetylimidazol (13 a).

Die bei der Neutralhydrolyse von $\mathbf{2 a}$ und $\mathbf{1 3}$ a ermittelten Hydrolysegeschwindigkeiten sprechen dafür, daß der zweite Reaktionsschritt, d.h. der Austritt von 4-Pyridon bzw. Imidazol, geschwindigkeitsbestimmend ist. Da sowohl bei 2 a als auch bei 13 a eine $N$-Acylbindung gespalten wird, sollte die Austrittstendenz mit der Acidität der korres- 
pondierenden Säure der Austrittsgruppe zunehmen ${ }^{12)}$. 4-Pyridon (1) $\left(\mathrm{p} K_{\mathrm{a} 1}=3.27^{13}\right)$ müßte demnach die bessere Austrittsgruppe als Imidazol $\left(\mathrm{p} K_{\mathrm{a} 1}=7.12^{14}\right)$ sein, was mit den Ergebnissen der Hydrolysegeschwindigkeiten übereinstimmt.

Das höhere Acylierungspotential von 2 a im Vergleich zu 13 a konnte auch präparativ durch direkte Umacylierung und durch kompetitive Acylierung bewiesen werden.

Löst man 2 a und Imidazol in Deuteriochloroform bei Raumtemperatur, so lassen sich schon nach wenigen Sekunden in der Lösung ${ }^{1} \mathrm{H}$-NMR-spektroskopisch ausschließlich 1 und $\mathbf{1 3 a}$ feststellen, d. h., die Umacylierung erfolgt sehr rasch und quantitativ.

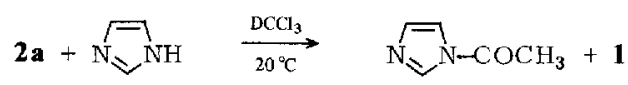

$13 \mathbf{a}$

Bei der Umsetzung äquimolarer Mengen 2a, 13a und Isopropylalkohol in Chloroform bei Raumtemperatur reagiert ausschließlich $\mathbf{2 a}$ mit dem Isopropylalkohol zu Essigsäure-isopropylester und 1, während $\mathbf{1 3}$ a unverändert erhalten bleibt, was wiederum ein Beweis für das größere Acylierungspotential von 2a verglichen mit 13a ist.

Bei der durch Alkoholat katalysierten Veresterung tertiärer Alkohole sind Imidazolide offensichtlich mindestens so reaktiv wie acylierte Pyridone ${ }^{3)}$.

Die Benzoylierung von tert-Butylalkohol mit 4-(Benzoyloxy)pyridin (3a) und 1-Benzoylimidazol in Tetrahydrofuran erfolgt mit dem Imidazolid rascher als mit 3a, in Methylenchlorid dagegen reagiert 3a schneller als 1-Benzoylimidazol, wobei jedoch bericksichtigt werden muß, daß keine homogenen Lösungen vorliegen.

Wir danken dem Fonds der Chemischen Industrie für die Unterstützung dieser Arbeit.

\section{Experimenteller Teil}

Acylierung von Aminen mit N-Acetyl- (2 a ${ }^{2)}$ und N-Formyl-4-pyridon (2 b) ${ }^{8)}$ bzw. 4-(Benzoyloxy)pyridin (3 a) 2): Zur Lösung von 2a, b oder $3 \mathbf{a}$ in absol. Methylenchlorid läßt man bei Raumtemp. unter Rühren rasch die äquimolare Menge Amin tropfen - hierbei erfolgt zum Teil Erwärmung - rührt die angegebene Zeit (Tab. 1) bei Raumtemp., destilliert das Methylenchlorid i. Vak. ab, extrahiert den Rückstand erschöpfend mit absol. Ether - hierbei bleibt 4-Pyridon (1) zurück - und reinigt den nach Abdampfen des Ethers erhaltenen Rückstand durch Vak.Destillation oder Umkristallisieren (Tab. 4).

Acylierung von Thiolen mit 2a und 3a: Die Umsetzungen werden wie bei der Acylierung von Aminen durchgeführt.

Acylierung primärer und sekundärer Alkohole mit $2 \mathrm{a}$ bzw. $3 \mathrm{a}$ in Methylenchlorid: Die Umsetzungen werden wie bei der Acylierung von Aminen durchgeführt (Tab. 6).

1-(4-Pyridyl)-4(IH)-pyridinon (11)

a) $2.74 \mathrm{~g}(20 \mathrm{mmol}) 2 \mathrm{a}$ werden in $50 \mathrm{ml}$ absol. 1,2-Dichlorethan im Bombenrohr 1 Woche auf $100^{\circ} \mathrm{C}$ erhitzt. Das Reaktionsgemisch wird filtriert, das Filtrat eingeengt und gekühlt und der ausgefallene braune Feststoff ( $1.3 \mathrm{~g})$ aus Benzol/Aktivkohle umkristallisiert. Ausb. $1.1 \mathrm{~g}(64 \%) 11$, Schmp. $180-181^{\circ} \mathrm{C}$ (Lit. ${ }^{45)} 177-179^{\circ} \mathrm{C}$ ).

b) Beim Erhitzen von 2 a bei 15 Torr destilliert in die gekühlte Vorlage Acetanhydrid (s. Lit. ${ }^{37}$ ), während ein Teil von $\mathbf{2 a}$ unzersetzt sublimiert. Der aus Benzol/Aktivkohle umkristallisierte Rückstand ist reines 11, Schmp. und Misch.-Schmp. $180-181^{\circ} \mathrm{C}$. 
Tab. 4. Acylierung von Aminen mit $\mathbf{2 a}$, $\mathbf{b}$ oder $\mathbf{3 a}$ bei Raumtemp. (Reaktionszeiten s. Tab. 1) in Methylenchlorid

\begin{tabular}{|c|c|c|c|c|c|c|c|}
\hline \multicolumn{2}{|c|}{$\begin{array}{l}\text { Acylierungs- } \\
\text { mittel } \\
\text { g (mmol) }\end{array}$} & \multirow{2}{*}{$\begin{array}{c}\underset{\mathrm{ml}}{\mathrm{CH}_{2} \mathrm{Cl}_{2}} \\
10\end{array}$} & \multirow{2}{*}{$\begin{array}{c}\begin{array}{c}\text { Amin } \\
\mathrm{g}(\mathrm{mmol})\end{array} \\
\begin{array}{c}0.99 \mathrm{a}) \\
(10.0)\end{array}\end{array}$} & \multicolumn{2}{|c|}{$\begin{array}{r}\text { Produkt } \\
\text { Ausb. } \\
\mathrm{g}(\%)\end{array}$} & \multirow[t]{2}{*}{$\begin{array}{c}\text { (Sdp. }{ }^{\circ} \mathrm{C} / \text { Torr) } \\
\text { Schmp. }{ }^{\circ} \mathrm{C}\end{array}$} & \multirow[t]{2}{*}{ Lit. } \\
\hline $2 a$ & $\begin{array}{l}1.37 \\
(10.0)\end{array}$ & & & $4 a$ & $\begin{array}{l}1.21 \\
(86)\end{array}$ & & \\
\hline $2 b$ & $\begin{array}{l}2.46 \\
(20.0)\end{array}$ & 50 & $\begin{array}{l}1.98 \text { a) } \\
(20.0)\end{array}$ & $5 \mathbf{a}$ & $\begin{array}{l}2.2 \\
(87)\end{array}$ & $(135-137 / 10)$ & $(140-142 / 10)^{16)}$ \\
\hline $\mathbf{3 a}$ & $\begin{array}{l}1.99 \\
(10.0)\end{array}$ & 10 & $\begin{array}{l}0.99 \text { a) } \\
(10.0)\end{array}$ & $6 a$ & $\begin{array}{l}1.81 \\
(89)\end{array}$ & $151-152$ & $149^{17)}$ \\
\hline $2 \mathfrak{a}$ & $\begin{array}{l}1.37 \\
(10.0)\end{array}$ & $15]$ & & $4 b$ & $\begin{array}{l}1.21 \\
(95)\end{array}$ & $131-132$ & $\begin{array}{l}(109 / 18)^{18)} \\
131-133^{19)}\end{array}$ \\
\hline $2 \mathbf{b}$ & $\begin{array}{l}1.23 \\
(10.0)\end{array}$ & 20 & $\begin{array}{l}0.85 \mathrm{~b}) \\
(10.0)\end{array}$ & $5 \mathbf{b}$ & $\begin{array}{l}0.95 \\
(84)\end{array}$ & $(100-102 / 12)$ & $(222 / 760)^{20)}$ \\
\hline $\mathbf{3 a}$ & $\begin{array}{l}1.99 \\
(10.0)\end{array}$ & 10 & & $6 \mathrm{~b}$ & $\begin{array}{l}1.75 \\
(93)\end{array}$ & $48-49$ & $49^{211}$ \\
\hline $2 \mathbf{a}$ & $\begin{array}{l}1.37 \\
(10.0)\end{array}$ & 157 & $0.61^{c)}$ & $4 c$ & $\begin{array}{l}0.87 \\
(85)\end{array}$ & $63-64$ (Aceton) & $63-65^{221}$ \\
\hline $2 b$ & $\begin{array}{l}1.23 \\
(10.0)\end{array}$ & 25 & $(10.0)$ & $5 c$ & $\begin{array}{l}0.71 \\
(80)\end{array}$ & $\left(108-109 / 10^{-3}\right)$ & $(191-193 / 10)^{23)}$ \\
\hline $2 a$ & $\begin{array}{l}1.37 \\
(10.0)\end{array}$ & $10)$ & & 4d & $\begin{array}{l}1.11 \\
(81)\end{array}$ & $112-114\left(\mathrm{H}_{2} \mathrm{O}\right)$ & $114^{24)}$ \\
\hline $2 \mathrm{~b}$ & $\begin{array}{l}1.23 \\
(10.0)\end{array}$ & 25 & $\begin{array}{l}0.93 \mathrm{~d}) \\
(10.0)\end{array}$ & $5 \mathrm{~d}$ & $\begin{array}{l}1.01 \\
(83)\end{array}$ & $\begin{array}{l}49-50 \text { (Petrolether/ } \\
\text { Xylol) }\end{array}$ & $50 ; 47^{25)}$ \\
\hline $\mathbf{3 a}$ & $\begin{array}{l}1.99 \\
(10.0)\end{array}$ & 10 & & $6 d$ & $\begin{array}{l}1.67 \\
(85)\end{array}$ & 163 (Ethanol) & $163 ; 160^{26)}$ \\
\hline $2 a$ & $\begin{array}{l}2.74 \\
(20.0)\end{array}$ & 30 & $\begin{array}{l}1.09 \mathrm{e}) \\
(10.0)\end{array}$ & f) & $\begin{array}{l}1.75 \\
(91)\end{array}$ & 98 & $98^{27)}$ \\
\hline
\end{tabular}

a) Cyclohexylamin. - b) Piperidin. - c) Ethanolamin. - d) Anilin. - e) 3-Aminophenol. f) 3-Acetoxy- $N$-acetylanilin.

Tab. 5. Acylierung von Thiolen (jeweils $10 \mathrm{mmol}) \mathrm{mit} 1.37 \mathrm{~g}(10 \mathrm{mmol}) 2 \mathrm{a}$ oder $1.99 \mathrm{~g}(10 \mathrm{mmol})$ 3 a bei Raumtemp. (Reaktionszeiten s. Tab. 2)

\begin{tabular}{|c|c|c|c|c|c|c|}
\hline $\begin{array}{l}\text { Acylie- } \\
\text { rungs- } \\
\text { mittel }\end{array}$ & $\begin{array}{c}\text { Lösungs- } \\
\text { mittel } \\
\text { ml }\end{array}$ & $\begin{array}{c}\text { Thiol } \\
\mathrm{g}\end{array}$ & & $\begin{array}{l}\text { dukt } \\
\text { Ausb. } \\
\text { g (\%) }\end{array}$ & $\begin{array}{l}\text { Sdp. }{ }^{\circ} \mathrm{C} / \mathrm{Torr} \\
\text { (Schmp. }{ }^{\circ} \mathrm{C} \text { ) }\end{array}$ & Lit. \\
\hline $\begin{array}{l}2 \mathbf{a} \\
\mathbf{3} \mathbf{a}\end{array}$ & $\begin{array}{l}10 \mathrm{CH}_{2} \mathrm{Cl}_{2} \\
10 \mathrm{CHCl}_{3}\end{array}$ & $\begin{array}{l}\text { 1-Butanthiol } \\
0.9\end{array}$ & $\begin{array}{l}7 a \\
8 a\end{array}$ & $\begin{array}{l}1.13(85) \\
1.70(89)\end{array}$ & $\begin{array}{l}134 \\
143 / 11\end{array}$ & $\begin{array}{l}134-135^{28)} \\
160 / 23^{29)}\end{array}$ \\
\hline $\left.\begin{array}{l}2 a \\
3 a\end{array}\right\}$ & $\begin{array}{l}10 \mathrm{CH}_{2} \mathrm{Cl}_{2} \\
10 \mathrm{CHCl}_{3}\end{array}$ & $\begin{array}{l}\text { Phenylmethanthiol } \\
1.24\end{array}$ & $\begin{array}{l}7 \mathbf{b}^{\text {a) }} \\
8 \mathrm{~b}\end{array}$ & $\begin{array}{l}1.46(88) \\
2.08(91)\end{array}$ & $\begin{array}{l}119-121 / 10 \\
(39)\end{array}$ & $\begin{array}{l}75.5-76.8 / \\
0.7-0.8^{30} \\
(39.5)^{31)}\end{array}$ \\
\hline $\left.\begin{array}{l}2 \mathbf{a} \\
\mathbf{3} \mathbf{a}\end{array}\right\}$ & $\begin{array}{l}10 \mathrm{CH}_{2} \mathrm{Cl}_{2} \\
10 \mathrm{CHCl}_{3}\end{array}$ & $\begin{array}{l}\text { Thiophenol } \\
1.10\end{array}$ & $\begin{array}{l}7 \mathrm{c} \\
8 \mathrm{c}\end{array}$ & $\begin{array}{l}1.32(87) \\
1.90(89)\end{array}$ & $\begin{array}{l}115-116 / 10 \\
(56)\end{array}$ & $\begin{array}{l}110-111 / 8^{28)} \\
(56-57)^{32)}\end{array}$ \\
\hline
\end{tabular}

a) $n_{\mathrm{D}}^{25}=1.5561$ (Lit, ${ }^{30)} 1.5565$ ). 
Tab. 6. Acylierung primärer und sekundärer Alkohole (jeweils $10 \mathrm{mmol})$ mit $1.37 \mathrm{~g}(10 \mathrm{mmol}) 2 \mathrm{a}$ oder $1.99 \mathrm{~g}(10 \mathrm{mmol}) 3 \mathrm{a}$ (Reaktionszeiten und -temp. s. Tab. 3) in Methylenchlorid

\begin{tabular}{|c|c|c|c|c|c|c|}
\hline $\begin{array}{l}\text { Acylie- } \\
\text { rungs- } \\
\text { mittel }\end{array}$ & $\underset{\mathrm{CH}_{2} \mathrm{Cl}_{2}}{\mathrm{ml}}$ & $\begin{array}{c}\text { Alkohol } \\
\mathrm{g}\end{array}$ & & $\begin{array}{l}\text { odukt } \\
\text { Ausb. } \\
\text { g(\%) }\end{array}$ & $\begin{array}{c}\text { Sdp, }{ }^{\circ} \mathrm{C} / \text { Torr } \\
\left(\text { Schmp. }{ }^{\circ} \mathrm{C}\right)\end{array}$ & Lit. \\
\hline $\begin{array}{l}\mathbf{2 a} \\
\mathbf{3 a}\end{array}$ & $\begin{array}{r}10 \\
5\end{array}$ & $\begin{array}{l}\text { Methanol } \\
0.32\end{array}$ & $\begin{array}{r}9 a \\
10 a\end{array}$ & 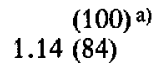 & $\begin{array}{l}57 \\
83 / 11\end{array}$ & $\begin{array}{l}57.1^{33)} \\
199.2^{34)}\end{array}$ \\
\hline $\begin{array}{l}\mathbf{2} \mathbf{a} \\
\mathbf{3} \mathbf{a}\end{array}$ & $\begin{array}{r}15 \\
5\end{array}$ & $\begin{array}{l}\text { 1-Butanol } \\
0.74\end{array}$ & $\begin{array}{r}9 b \\
10 b\end{array}$ & $\begin{array}{l}1.05(91) \\
1.50(84)\end{array}$ & $\begin{array}{l}125 \\
136-137 / 11\end{array}$ & $\begin{array}{l}126.1^{35)} \\
250.3^{36)}\end{array}$ \\
\hline $\begin{array}{l}\mathbf{2 a} \\
\mathbf{3} \mathbf{a}\end{array}$ & $\begin{array}{r}10 \\
5\end{array}$ & $\begin{array}{l}\text { Benzylalkohol } \\
1.08\end{array}$ & $\begin{array}{r}9 c \\
10 c\end{array}$ & $\begin{array}{l}1.3(87) \\
1.86(88)\end{array}$ & $\begin{array}{l}98 / 12 \\
178-179 / 12\end{array}$ & $\begin{array}{l}\left.93-94 / 10^{37}\right) \\
170-171 / 11^{39)}\end{array}$ \\
\hline $\begin{array}{l}2 \mathbf{a} \\
3 \mathbf{a}\end{array}$ & $\begin{array}{r}15 \\
5\end{array}$ & $\begin{array}{l}\text { Allylalkohol } \\
0.58\end{array}$ & $\begin{array}{r}9 \mathrm{~d} \\
10 \mathrm{~d}\end{array}$ & $\begin{array}{l}0.91(91) \\
1.28(79)\end{array}$ & $\begin{array}{l}102-103 \\
128-129 / 11\end{array}$ & $\begin{array}{l}103.5 / 760 \\
\left.242 / 228^{40}\right)\end{array}$ \\
\hline $\begin{array}{l}2 \mathbf{a} \\
\mathbf{3} \mathbf{a}\end{array}$ & $\begin{array}{r}10 \\
5\end{array}$ & $\begin{array}{l}\text { Phenol } \\
0.94\end{array}$ & $\begin{array}{r}9 \mathrm{e} \\
10 \mathrm{e}\end{array}$ & $\begin{array}{l}1.17(86) \\
1.74(88)\end{array}$ & $\begin{array}{l}85-86 / 12 \\
(70-71)\end{array}$ & $\begin{array}{l}195.7 ; 75-76 / 8^{41)} \\
(69 ; 71)^{42)}\end{array}$ \\
\hline $\begin{array}{l}\mathbf{2} \mathbf{a} \\
\mathbf{3} \mathbf{a}\end{array}$ & $\begin{array}{r}15 \\
5\end{array}$ & $\begin{array}{l}\text { Cyciohexanol } \\
10.0\end{array}$ & $\begin{array}{r}9 \mathrm{f} \\
10 \mathrm{f}\end{array}$ & $\begin{array}{l}1.23(87) \\
1.65(81)\end{array}$ & $\begin{array}{l}62-63 / 12 \\
152-153 / 11\end{array}$ & $\begin{array}{l}173 ; 63 / 12^{37)} \\
285^{38)}\end{array}$ \\
\hline $2 a$ & 10 & $\begin{array}{l}\text { Isopropyl- } \\
\text { alkohol } \\
0.60\end{array}$ & $9 \mathrm{~g}$ & $\begin{array}{l}(100)^{\text {a) }} \\
0.77(76)\end{array}$ & $\begin{array}{l}89-90 \\
91-92\end{array}$ & $\begin{array}{l}88.9 ; 91^{33)} \\
218 ; 543\end{array}$ \\
\hline $\begin{array}{l}\mathbf{3} \mathbf{a} \\
2 \mathbf{a}\end{array}$ & 5 & 0.60 & $10 \mathrm{~g}$ & $1.39(85)$ & $\begin{array}{l}91-92 / 11 \\
\end{array}$ & $\begin{array}{l}\left.218.5^{43}\right) \\
\left(115-(16)^{44)}\right.\end{array}$ \\
\hline $\begin{array}{l}2 \mathbf{a} \\
\mathbf{3} \mathbf{a}\end{array}$ & $\begin{array}{l}30 \\
20\end{array}$ & $\begin{array}{l}\text { Cholesterin } \\
3.86\end{array}$ & $\begin{array}{r}9 \mathrm{~h} \\
10 \mathrm{~h}\end{array}$ & $\begin{array}{l}4.12(96) \\
4.36(89)\end{array}$ & $\begin{array}{l}(115) \\
(149-151)\end{array}$ & $\begin{array}{l}(115-116)^{44)} \\
(152-153)^{44)}\end{array}$ \\
\hline
\end{tabular}

a) Gaschromatographisch und über ${ }^{1} \mathrm{H}$-NMR-Integral bestimmt.

c) 60 stdg. Erhitzen von 2 a auf $150^{\circ} \mathrm{C}$ ergibt quantitativ 11 und Acetanhydrid, wie der ${ }^{1} \mathbf{H}$ NMR-spektroskopische Vergleich mit authentischen Substanzen ergab.

Essigsäure-tert-butylester $(\mathbf{1 2}, \mathrm{X}=\mathrm{H})$ : Wie bei der Acylierung primärer und sekundärer Alkohole mit $2 \mathrm{a}$ oder $3 \mathrm{a}$ beschrieben, aus $1.37 \mathrm{~g}(10.0 \mathrm{mmol}) \mathbf{2 a}$ und $0.74 \mathrm{~g}(10.0 \mathrm{mmol})$ tertButylalkohol in $15 \mathrm{ml}$ 1,2-Dichlorethan nach $3 \mathrm{~d}$ Erhitzen unter Rückfluß Ausb. 35\% (gaschromatographisch bestimmt) ${ }^{46)}$.

Benzoesäure-tert-butylester $\left(10, \mathrm{R}^{1}=\mathrm{C}\left(\mathrm{CH}_{3}\right)_{3}\right)$ : Zur Lösung von $83.0 \mathrm{mg}(3.6 \mathrm{mmol})$ Natrium

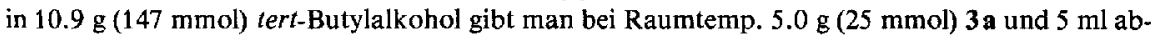
sol. Methylenchlorid, rührt $4 \mathrm{~d}$ bei Raumtemp., entfernt vom Lösungsmittel und überschüssigem Alkohol i. Vak., versetzt den Rückstand mit je $50 \mathrm{ml}$ Wasser und Ether, läßt $2 \mathrm{~h}$ stehen, schüttelt die wäßr. Schicht dreimal mit Ether aus und die vereinigten Etherextrakte mit $2 \mathrm{~N}$ wäßr. Natriumcarbonatlösung. Nach Trocknen über Natriumsulfat, Entfernen des Ethers und Destillieren des Rückstandes erhält man $4.0 \mathrm{~g}(90 \%) 10\left(\mathrm{R}^{1}=\mathrm{C}\left(\mathrm{CH}_{3}\right)_{3}\right)$, Sdp. $96^{\circ} \mathrm{C} / 12$ Torr, $[\alpha]_{\mathrm{D}}^{20}=1.4907$ (Lit. ${ }^{47)}$ Sdp. $96^{\circ} \mathrm{C} / 2$ Torr).

Trichloressigsäure-tert-butylester (12 b): Aus $4.8 \mathrm{~g}$ (20.0 mmol) 4-(Trichloracetoxy)pyridin ${ }^{2)}$ in $30 \mathrm{ml}$ absol. Methylenchlorid und $1.48 \mathrm{~g}(20.0 \mathrm{mmol})$ tert-Butylalkohol erhält man nach $3 \mathrm{~h}$ Reaktionszeit bei $20^{\circ} \mathrm{C}$ und Aufarbeiten, wie bei der Acylierung mit Alkoholen beschrieben, $2.0 \mathrm{~g}$ (45\%) 12 b, Sdp. $63-64^{\circ} \mathrm{C} / 12$ Torr (Lit. 48$) 37^{\circ} \mathrm{C} / 1$ Torr), Schmp. $25.5^{\circ} \mathrm{C}$.

Trifluoressigsäure-tert-butylester (12 c): Aus $1.9 \mathrm{~g}$ (10.0 mmol) 4-(Trifluoracetoxy)pyridin ${ }^{2)}$ in $15 \mathrm{ml}$ absol. Methylenchlorid und $0.74 \mathrm{~g}(10.0 \mathrm{mmol})$ tert-Butylalkohol wie vorstehend. Ausb. $1.46 \mathrm{~g}(86 \%)$, Sdp. $83^{\circ} \mathrm{C}$ (Lit. $4^{49)} 83^{\circ} \mathrm{C} / 760$ Torr).

Konduktometrische Bestimmung der Hydrolysegeschwindigkeit von $\mathbf{2 a}$ und 13 a: Gerät: Metrohm Konduktoskop E 365 B, Elektrode Philips PW 9512/00, Zellfaktor $(f) 1.34 \mathrm{~cm}$, Zellkon- 
stante $c=1 / f=0.746 \mathrm{~cm}^{-1}$. Leilfähigkeit des Leitfähigkeitswassers zwischen 0.5 und $1.10^{-6}$ $\Omega^{-1} \mathrm{~cm}^{-1}$. Konzentration $1 \mathrm{mg}$ Substanz in $10 \mathrm{ml}$ Leitfähigkeitswasser. Temperatur $23^{\circ} \mathrm{C} \pm 1$.

Umacylierung: $137 \mathrm{mg}(1.0 \mathrm{mmol}) 2 \mathrm{a}$ und $68 \mathrm{mg}(1.0 \mathrm{mmol})$ Imidazol werden in $1 \mathrm{ml}$ Deuteriochloroform bei Raumtemp. gelöst, und sofort wird ${ }^{1}$ H-NMR-spektroskopisch untersucht. Es erscheinen lediglich die Signale von 1-Acetylimidazol (13 a) und 4-Pyridon (1).

Kompetitive Veresterung von Isopropylalkohol mit 2 a und 1-Acetylimidazol (13a): Die Lösung von $137 \mathrm{mg}(1.0 \mathrm{~mol}) 2 \mathrm{a}, 110 \mathrm{~g}(1.0 \mathrm{mmol}) 13 \mathrm{a}$ und $60 \mathrm{mg}(1.0 \mathrm{mmol})$ Isopropylalkohol in $1.5 \mathrm{ml}$ Deuteriochloroform wird nach 40 h Stehenlassen bei Raumtemp. ${ }^{1}$ H-NMR-spektroskopisch untersucht. Es erscheinen die Signale von 1, Essigsäure-isopropylester und 13a.

Vergleichende Benzoylierung von tert-Butylalkohol mit 3 a und 1-Benzoylimidazol: Die Umsetzungen werden durchgeführt, wie bei der Darstellung von Benzoesäure-tert-butylester $\left(\mathbf{1 0}, \mathbf{R}^{1}=\right.$ $\left.\mathrm{C}\left(\mathrm{CH}_{3}\right)_{3}\right)$ beschrieben (Tab. 7).

Tab. 7. Vergleichende Benzoylierung von $15.0 \mathrm{~g}$ (202 mmol) tert-Butylalkohol mit $6.8 \mathrm{~g}$ (34 mmol) $3 \mathrm{a}$ bzw. $5.9 \mathrm{~g}$ (34 mmol) 1-Benzoylimidazol in Gegenwart von $115 \mathrm{mg} \mathrm{(5} \mathrm{mmol)}$ Natrium in $10 \mathrm{ml}$ Lösungsmittel, Reaktionsdauer $16 \mathrm{~h}$ bei $20^{\circ} \mathrm{C}$

\begin{tabular}{|c|c|c|c|}
\hline \multirow{2}{*}{$\begin{array}{l}\text { Acylierungs- } \\
\text { mittel }\end{array}$} & \multirow{2}{*}{$\begin{array}{l}\text { Lösungs- } \\
\text { mittel }\end{array}$} & \multicolumn{2}{|c|}{ Ausb. Benzoesäure-tert-butylester } \\
\hline & & $\mathrm{g}$ & $\%$ \\
\hline $3 \mathbf{a}$ & THF & & $18^{a)}$ \\
\hline 1-Benzoylimidazol & THF & 3.93 & 65 \\
\hline $3 \mathbf{a}$ & $\mathrm{CH}_{2} \mathrm{Cl}_{2}$ & & $23^{a)}$ \\
\hline 1-Benzoylimidazol & $\mathrm{CH}_{2} \mathrm{Cl}_{2}$ & & $2^{\text {a) }}$ \\
\hline
\end{tabular}

a) Gaschromatographisch bestimmt.

\section{Literatur}

1) Teil der Dissertation $E$. Bessey, Univ. Stuttgart 1977.

2) F. Effenberger, A. O. Mück und E. Bessey, Chem. Ber. 113, 2086 (1980), vorstehend.

3) 3a) $A$. H. Staab und $W$. Rohr in Neuere Methoden der präparativen organischen Chemie, Bd. V, S. 53, Verlag Chemie, Weinheim/Bergstraße 1967. - 3b) H. J. Gais, Angew. Chem. 89, 251 (1977); Angew. Chem., Int. Ed. Engl. 16, 244 (1977).

4) Y. Ueno, T. Takaya und E. Imoto, Bull. Chem. Soc. Jpn. 37, 864 (1964).

5) A. S. Dutta und J. S. Morley, J. Chem. Soc. C 1971, 2896.

6) 6a) V. A. Savelova, A. V. Skripka und L. M. Litvinenko, Zh. Org. Khim. 14, 1260 (1978) [Chem. Abstr. 89, $89864 \mathrm{t}$ (1978)]. - 6b) J. F. Carson, Synthesis 1979, 24.

7) $P$. A. Singgih und M. J. Janssen, Tetrahedron Lett. 1971, 4223.

8) F. Effenberger, M. Keil und E. Bessey, Chem. Ber. 113, 2110 (1980), nachstehend.

9) J. P. Wibaut und F. W. Broekman, Rec. Trav. Chim. Pays-Bas 78, 593 (1959).

10) H.S. Isaacs, Reactive Intermediates in Organic Chemistry, S. 422, J. Wiley and Sons, London-New York-Sydney-Toronto 1974.

11) H. A. Staab, Chem. Ber. 89, 1939 (1956).

12) E. A. Castro und F. J. Gil, J. Am. Chem. Soc. 1977, 7611; dort weitere Literaturhinweise.

13) A. Albert und J. N. Phillips, J. Chem. Soc. 1956, 1294.

14) P. Brooks und N. Davidson, J. Am. Chem. Soc. 82, 2118 (1960).

15) Z. Rappoport, Handbook of Tables of Organic Compound Identification, 3. Aufl., S. 234, The Chemical Rubber Co., Cleveland, Ohio 1967.

16) $H$. Wieland und E. Dorrer, Ber. Dtsch. Chem. Ges. 63 (B), 404 (1930).

17) Lit. 15), S. 238.

18) R. C. Weast, Handbook of Chemistry and Physics, 57 Ed. C-441, The Chemical Rubber Co., Cleveland, Ohio 1976-1977.

19) Lit. ${ }^{18)}$, jedoch 53. Ed., $1972-1973$, C-435. 
20) Lit. ${ }^{15)}$, S. 230.

21) Lit. ${ }^{18)}, \mathrm{C}-441$.

22) Lit. ${ }^{18)}, \mathrm{C}-84$.

23) H. Wenker, J. Am. Chem. Soc. 57, 1079 (1935).

24) Lit. 15), S. 235.

25) Lit. 15), S. 231.

26) Lit. ${ }^{15)}$, S. 240.

27) $M$. Julia und $M$. Baillarǵe, Bull. Soc. Chim. Fr. 1952, 639.

28) A. W. Ralston und J. A. Wilkinson, J. Am. Chern. Soc. 50, 2162 (1928).

29) J. W. Kimball und E. E. Ried, J. Am. Chem. Soc. 38, 2757 (1916).

30) B. K. Morse und D. S. Tarbell, J. Am. Chem. Soc. 74, 416 (1952).

31) E. Fromm und A. Forster, Liebigs Ann. Chem. 394, 339 (1912).

32) Y. Kanaoka, K. Tanizawa, E. Sato, O. Yonemitsu und Y. Ban, Chem. Pharm. Bull. (Tokyo) 15, 593 (1967) (Eng.) [Chem. Abstr. 67, 63996h (1967)].

33) Lit. 15), S. 250.

34) Lit. 15), S. 258.

35) Lit. ${ }^{15)}$, S. 252

36) Lit. 15$)$, S. 266.

37) Lit. ${ }^{19)}, \mathrm{C}-80$.

38) Lit. 19), C-175.

39) Lit, 19), C-176.

40) Lit. ${ }^{18)}, \mathrm{C}-180$.

41) Lit. 19), C-82.

42) Lit. ${ }^{15)}$, S. 277.

43) Lit. 15), S. 261.

44) Lit. 19), C-240.

45) $P$. A. de Villiers und $H$. J. den Hertog, Rec. Trav. Chim. Pays-Bas 76, 647 (1957).

46) Lit. ${ }^{18)}$, C-86.

47) D. F. Norris und G. W. Rigby, J. Am. Chem. Soc. 54, 2088 (1932).

48) W. E. Scovill, R. E. Burk und H. P. Lankelma, J. Am. Chem. Soc. 66, 1039 (1944).

49) $M . M$. Jouillé, J. Am. Chem. Soc. 77, 6662 (1955). 